\title{
Lipoblast-Like Morphology in a Uveal Melanoma with Delayed Metastasis to the Liver
}

\author{
Aimi Toyama W. Robert Bell Alessio Giubellino Faqian Li \\ Department of Laboratory Medicine and Pathology, University of Minnesota, Minneapolis, MN, USA
}

\section{Established Facts}

- Uveal melanomas often metastasize to the liver.

- Latent melanomas are more common and occur after a longer period of time than cutaneous melanomas.

- Common cell morphologies in uveal melanomas include epithelioid and spindle.

- S100 negativity and unusual morphology make the diagnosis of metastatic uveal melanoma difficult.

\section{Novel Insights}

- Lipoblast-like uveal melanoma can mimic lipogenic tumors or benign steatotic change upon metastasis to the liver.

\section{Keywords}

Latent melanomas · Lipoblast · Liver · Melanoma ·

Pathology · S100 · Signet ring · Tumors · Uveal melanoma

\begin{abstract}
Uveal melanomas are typically described as having epithelial or spindled cell morphology; however, as is the nature of melanomas, the morphology of the malignant melanocytes can be varied. We describe a unique case of metastatic uveal melanoma with lipoblast-like morphology, identified in the liver during an autopsy of a 75-year-old woman. Apart from a remote history of uveal melanoma in the right eye, there was no other history of cancer, and there were no con-
\end{abstract}

cerning skin lesions present. The liver exhibited hepatomegaly with diffuse and extensive involvement of malignant tumor cells. Histopathological evaluation of liver sections showed malignant epithelioid and spindle cell proliferation. A distinct, spiculated, tan-white area revealed sheets of malignant cells with small and large vacuoles within the cytoplasm and scalloped nuclei, mimicking lipoblasts and adipocytes. Immunohistochemical stains confirmed these cells to be malignant melanoma cells. Being aware of this morphology in uveal melanomas is important especially when there is metastasis to the liver, so that it is not mistaken for more benign processes such as steatosis.

(c) 2019 S. Karger AG, Basel

\section{KARGER}

(c) 2019 S. Karger AG, Basel

E-Mail karger@karger.com

www.karger.com/oop
Aimi Toyama, MD

Department of Laboratory Medicine and Pathology

University of Minnesota, Mayo Mail Code 609, 420 Delaware Street S.E.

Minneapolis, MN 55455 (USA)

E-Mail toyama@umn.edu 


\section{Introduction}

Lipoblast-like cells are unusual in melanomas, and they are seldom described as a morphologic feature of uveal melanomas owing to its rarity. When observed as a metastasis, this morphology is strikingly similar to liver lesions with steatosis or lipogenic tumors. We describe a case of metastatic uveal melanoma with lipoblast-like morphology after a long latency, mimicking a primary liver malignancy.

\section{Case Presentation}

We present a case of a 75-year-old woman who visited the Emergency Department due to progressive weakness, dyspnea on exertion, and intermittent loose and bloody stools, as well as unexplained weight loss of more than $30 \mathrm{lbs}$ over a 7 -month period. Vital signs were stable, but physical examination showed massive hepatomegaly. A non-contrast CT as well as an ultrasound were performed, revealing a markedly enlarged liver measuring up to $27.1 \mathrm{~cm}$ in length with coarsened echogenicity, suspicious of an infiltrative tumor (Fig. 1). Laboratory results showed elevated alkaline phosphatase (271 U/L, reference range 40-150 U/L), AST (144 U/L, reference range $0-45 \mathrm{U} / \mathrm{L})$, and total bilirubin $(3.0 \mathrm{mg} /$ $\mathrm{dL}$, reference range $0.2-1.3 \mathrm{mg} / \mathrm{dL}$ ) levels, with a normal ALT. The patient's past medical history was remarkable for a uveal melano- ma on the right eye, 15 years ago, which was diagnosed and treated with local radiation and cryotherapy. She had no other history of known cancers. A biopsy of the liver was scheduled; however, before it could be performed, the patient decompensated and died. Thus, an autopsy was requested.

Upon initial examination of the internal organs, the hepatomegaly was striking, as it obstructed the view of approximately $90 \%$ of the bowel. The liver weighed 4,480 $\mathrm{g}$ and had a bumpy and mottled surface. Sectioning revealed variably sized nodules with black, tan-white, and light pink discoloration (Fig. 2a). Grossly conspicuous was a $2.0 \times 1.4 \mathrm{~cm}$ tan area that was firmer and had a spiculated and irregular outline (Fig. 2a, b). Hematoxylin and eosin sections of the liver showed diffuse infiltration of the hepatic parenchyma by malignant spindle and epithelioid tumor cells with intracellular melanocytic pigment and melanophages (Fig. 2c). The hepatocytes adjacent and away from the metastatic melanoma demonstrated only focal mild steatosis without significant inflammation or fibrosis. Within the focal area that was different from the infiltrating malignant spindle and epithelioid cells, there were sheets of tumor cells characterized by small and large intracytoplasmic vacuoles with the nucleus pushed to the side, resembling lipoblasts and adipocytes (Fig. 3). Immunohistochemical staining revealed the malignant spindle and epithelioid cells to be positive for S100, Melan-A, SOX10, HMB-45, and negative for cytokeratin AE1/AE3. The lipoblast- and adipocyte-like cells, however, were negative for S100, yet positive for all other melanocytic markers (Fig. 3). Cytokeratin AE1/AE3 was negative in these cells (Fig. 3), ruling out a benign incidental hepatocellular adenoma or an area of steatotic hepatocyte change.
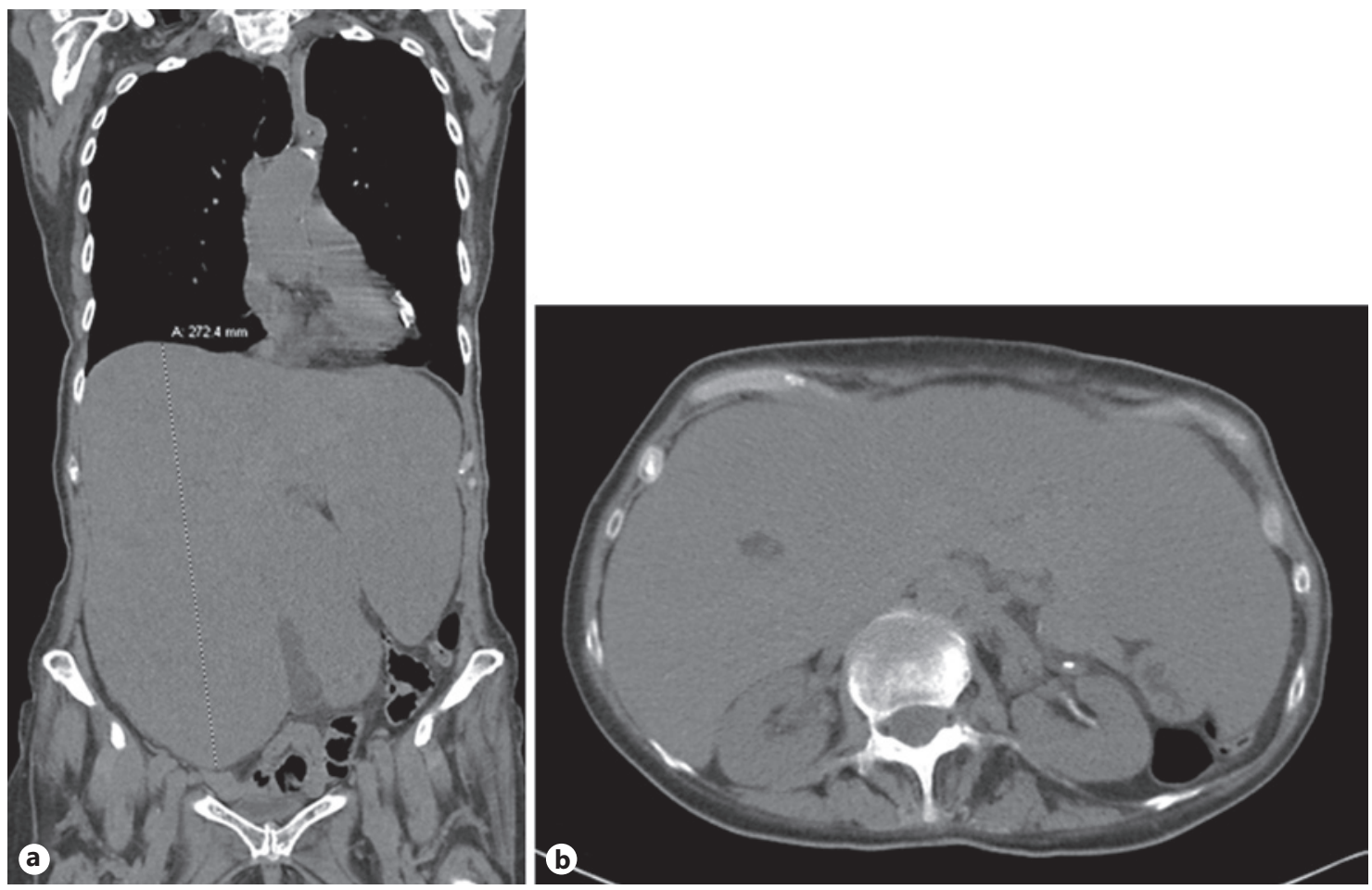

Fig. 1. a CT imaging reveals severe hepatomegaly. b A small hypoenhancing area is seen in the right side of the enlarged liver. 
The eyes themselves were not removed for examination as it was not standard procedure for autopsies and there was no note of visual disturbances or pertinent eye findings on physical exam upon review of the medical records. With the absence of any other known history of cutaneous or mucosal melanomas and no concerning skin lesions identified during the autopsy, we believed that the patient's metastatic melanoma was a recurrence of her remote uveal melanoma. To support a uveal origin, an immunohistochemical stain testing for expression of BRCA1-associated protein 1 (BAP1) was performed, which has been found to frequently be lost in uveal melanomas [1]. In our case, BAP1 was negative in the tumor cells but positive in surrounding benign liver cells, further suggesting a uveal origin for the metastatic melanoma (Fig. 2d).

\section{Discussion/Conclusion}

Between the iris, ciliary body, and the choroid that make up the uvea, the choroid is the most common origin of uveal melanomas [2]. Up to $90 \%$ of metastatic cases involve the liver, but other sites of involvement can include the lung, bone, and skin [3]. In our case, in addition to the malignant melanoma cells found diffusely in the liver, there were also multiple microscopic foci of meta- static epithelioid melanoma without lipoblast-like morphology present in the lungs. In contrast, in cutaneous melanomas, when distant metastases occur, they are more commonly detected in the skin, subcutaneous tissue, and distant lymph nodes [4]. The liver is estimated to be involved only $14-20 \%$ of the time when detected by clinical and imaging techniques [4].

In our case, the patient had a 15-year latency from the time of diagnosis to the time of having reported clinical symptoms of recurrence. Late recurrences that are defined as occurring 10 years or more after diagnosis and subsequent treatment are unusual in melanoma, but studies have shown that they more commonly occur in ocular or uveal melanomas as compared to cases of primary cutaneous melanoma [5]. In a study of 168 patients with late recurrence melanoma, Crowley and Seigler [5] found the mean time to recurrence for the 155 cases of cutaneous melanomas to be 14.3 years, while it was 22.3 years for the 12 cases of ocular melanomas. Furthermore, they found that ocular melanomas had a statistically significantly shorter survival period after recurrence when compared to cutaneous melanomas; the patient in our
Fig. 2. a Cut surface of the liver showing diffuse replacement of parenchyma by variably sized and discolored nodules as well as a focal tan, spiculated area (arrow) that appeared different from the surrounding nodules. b Low-power view of the section of the aforementioned area with tumor nodules. $\mathrm{H} \& \mathrm{E}, \times 0.4$. c Area showing transition from epithelioid morphology (bottom left) into lipoblast-like morphology (right), with hepatocytes percolating in between. $\times 20$. d BAP1 stained negative in the tumor cells and positive in the benign hepatocytes, supporting a uveal origin. $\times 20$.
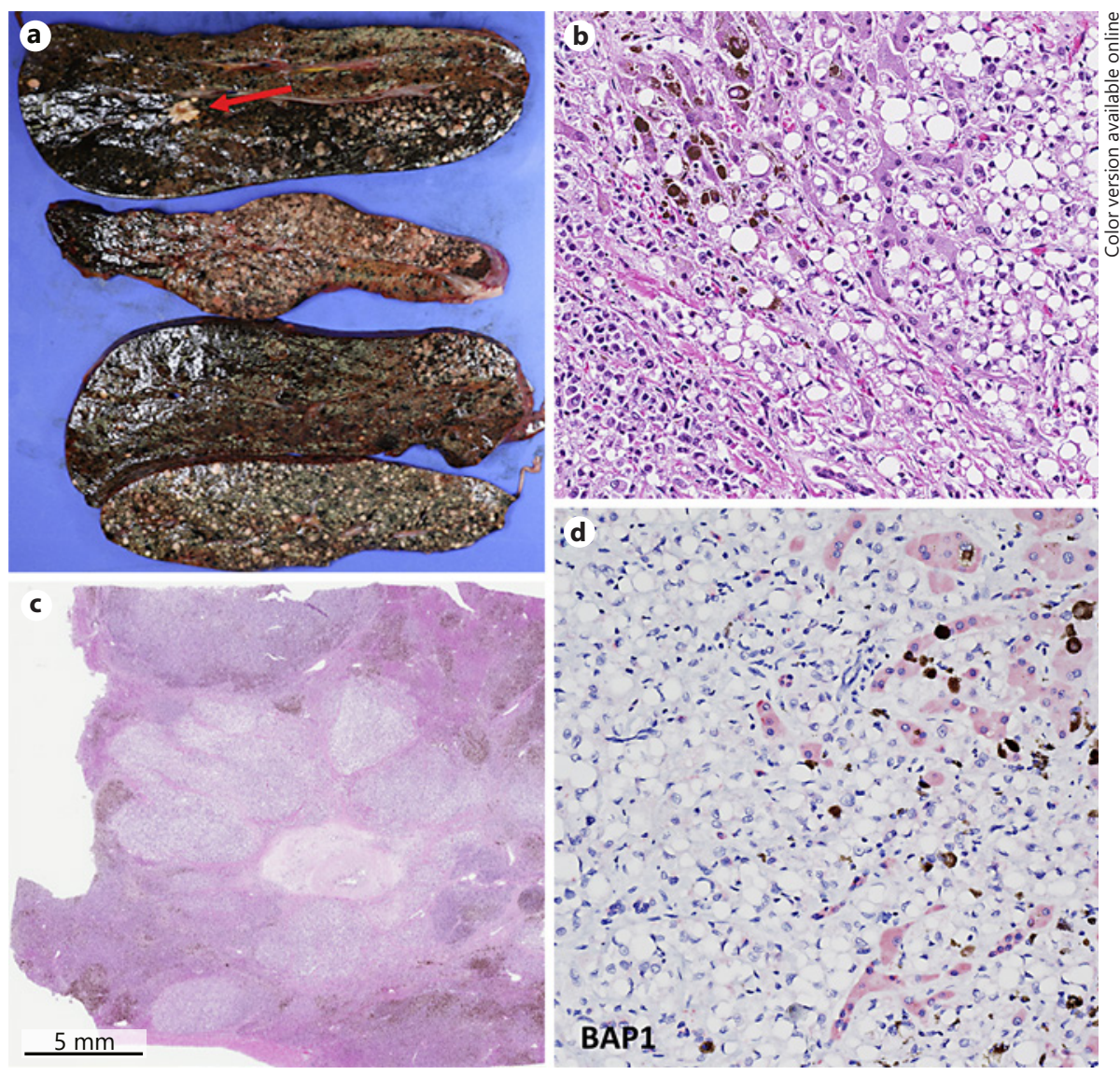

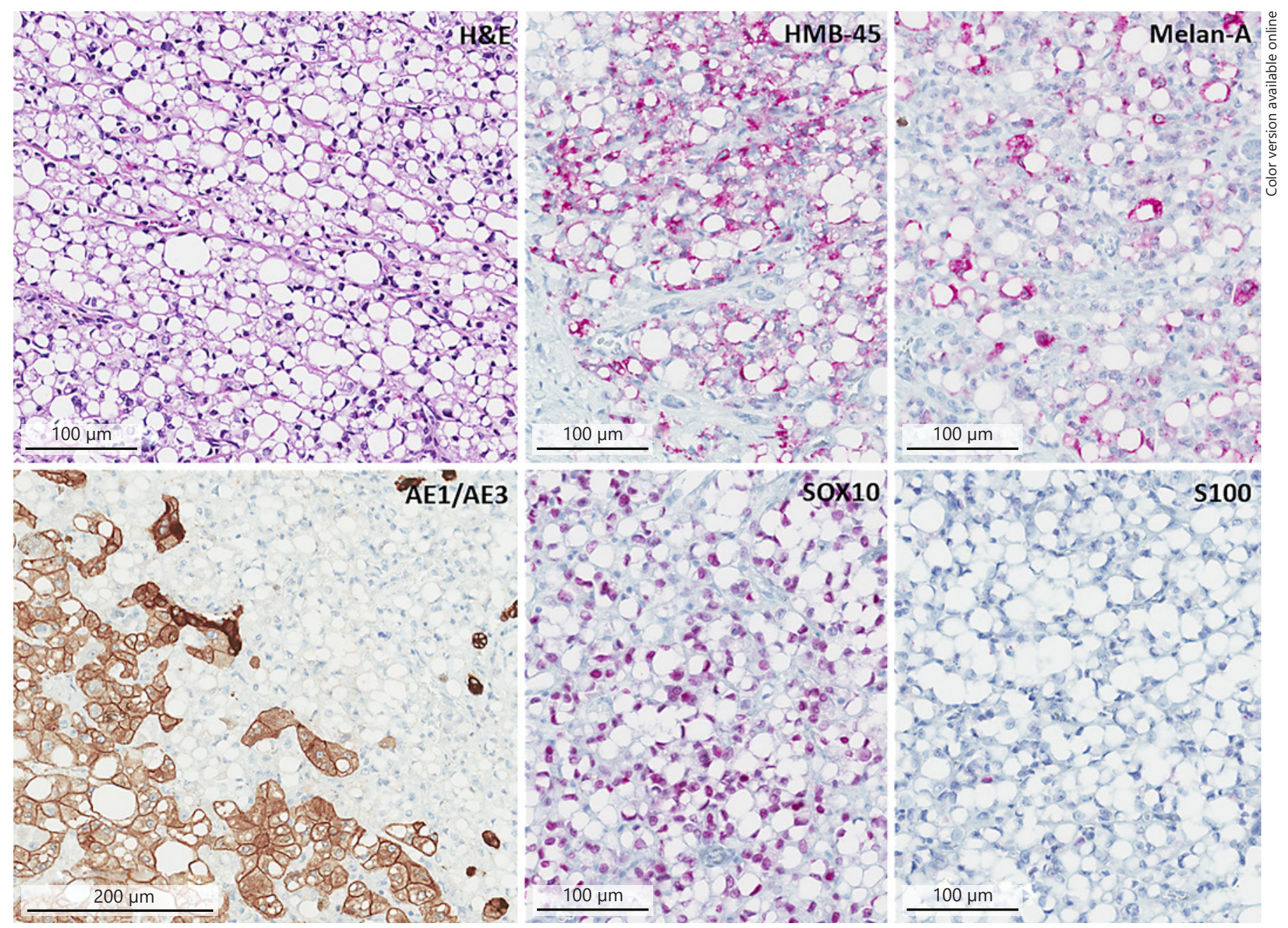

Fig. 3. Malignant cells contain large, cleared-out cytoplasmic vacuoles that push the nucleus to the side and distort its shape, resembling lipoblasts and adipocytes. The immunohistochemical melanoma panel performed in this area showed positive staining for HMB-45, Melan-A, and SOX10, but was negative for S100 and cytokeratin AE1/AE3. The uninvolved hepatocytes stain positively for AE1/AE3.

case died only 3 months after showing initial symptoms of recurrence [5].

The histopathologic finding of lipoblast-like cells in cutaneous melanoma was most recently described in a case report in 2002 by Cruz et al. [6]. They described pleomorphic cells with large cleared-out vacuoles resembling lipid droplets that displace the cytoplasm and push the nucleus to the periphery, as well as cells with a central stellate nuclei and multiple small clear vacuoles around the periphery [6]. Such cells have been previously reported as variants of signet ring cell melanomas $[6,7]$. Both signet ring cells and lipoblast-like cells in melanoma likely represent different degrees of cytoplasmic vacuolar change. In our case, the majority of vacu- olated cells were morphologically more akin to lipoblasts and adipocytes than signet ring cells. The typical cell types that are seen in uveal melanomas are spindle and epithelioid, with the epithelioid cell predominant type having a worse prognosis $[8,9]$. Signet ring type morphology in uveal melanoma is an entity not commonly mentioned in the literature, and there have not been many cases since being first described in 1996 [10]. In a 2014 case series of signet ring cell melanomas of the skin by Kocovski and Alowami [11], they reported only 23 cases, and there have only been few other cases reported since then, with only a single abstract documenting lipoblast-like melanoma originating from the ciliary body $[7,11]$. Thus, to the best of our knowledge, this is 
one of only few cases described in the literature of lipoblast-like morphology in uveal melanoma.

To aid and supplement the diagnosis of melanoma, immunohistochemical stains are essential. Since being first introduced as a possible melanoma marker by Gaynor et al. [12] in 1980, S100 has been invaluable to pathologists due to its sensitivity for melanocytes. When there is strong and diffuse staining of the cells of interest with S100, melanoma is surely unable to be excluded from any differential diagnosis. According to a recent review of the literature on melanocyte-associated immunohistochemical stains, the sensitivity of the $S 100$ stain was very high, ranging from approximately 93 to $100 \%$ [13]. However, specifically in uveal melanomas, S100 staining may not be as helpful. When comparing the staining pattern differences between cutaneous and uveal melanomas for various stains including S100, Iwamoto et al. [8] found that whereas $S 100$ showed strong positivity in cutaneous melanomas, the staining was only weak and variable for uveal melanomas. Melan-A, tyrosinase, HMB-45, HMB50 , and MITF were instead recommended for identification of uveal melanomas over the S100 stain [8]. Curiously, in our case, even though the background metastatic melanocytes in the liver stained positively for S100, the areas with lipoblast-like cells showed absent S100 staining. While other authors have suggested the morphologic changes of these malignant cells may be attributed to degenerative change, this could perhaps be owing to the variability of $S 100$ staining in uveal melanomas as previously mentioned, or it may suggest that these malignant cells have undergone true metaplastic change [14]. SOX10, a nuclear transcription factor and another useful marker of neural crest-derived cells involved in maintenance of Schwannian and melanocytic cell differentiation, was retained in the lipoblast-like malignant cells. A study by Nonaka et al. [15] compared the staining patterns of SOX10 and S100 in cutaneous melanomas, and SOX10 was found to be a more sensitive melanoma marker than S100, with $97 \%$ of melanomas staining positive for SOX10 and 91\% staining positive for S100.

Signet ring type melanomas are rare, but the lipoblastlike morphology in melanomas is even rarer, especially in uveal melanomas. Because uveal melanoma preferentially metastasizes to the liver, it is important to be aware of the existence of this morphology so as to not mistake them for benign entities. Fatty, steatotic change within the liver as well as hepatocellular adenomas can show similar features with cytoplasmic vacuoles and scalloped nuclei; however, cytokeratin stains will be positive and can help distinguish this from metastatic melanoma. S100 may not be as useful in identifying cases of uveal melanoma; however, performing other melanoma markers such as Melan-A, HMB-45, or tyrosinase can help to rule in this unfamiliar diagnosis.

\section{Statement of Ethics}

Consent for autopsy including permission for research was obtained from the next of kin. The research protocol for human subjects was approved by Institutional Review Boards at the University of Minnesota. We have de-identified the patient to the best of our ability.

\section{Disclosure Statement}

The authors have no conflicts of interest to declare.

\section{Funding Sources}

The authors received no funding for this paper.

\section{Author Contributions}

Aimi Toyama, MD - primary author and prosector of autopsy, acquired gross and microscopic images, approved final publishable version, and agreed to be accountable for all aspects of the work.

W. Robert Bell, MD, MHS - contributed to interpretation of data, revised for important intellectual content, approved final publishable version, and agreed to be accountable for all aspects of the work.

Alessio Giubellino, $\mathrm{MD}, \mathrm{PhD}$ - contributed to staining and interpretation of BAP1 immunohistochemical stain, approved final publishable version, and agreed to be accountable for all aspects of the work.

Faqian Li, MD, PhD - contributed to conception, acquisition, and interpretation of the case; revised for important intellectual content; approved final publishable version; and agreed to be accountable for all aspects of the work.
References
1 Harbour JW, Onken MD, Roberson ED, Duan S, Cao L, Worley LA, et al. Frequent mutation of BAP1 in metastasizing uveal mel- anomas. Science. 2010 Dec;330(6009): $1410-3$.
2 Damato B, Singh AD, editors. Clinical oph- thalmic oncology: uveal tumors. Heidelberg: Springer; 2014.
3 Spagnolo F, Caltabiano G, Queirolo P. Uveal melanoma. Cancer Treat Rev. 2012 Aug; 38(5):549-53. 
4 Leiter U, Meier F, Schittek B, Garbe C. The natural course of cutaneous melanoma. J Surg Oncol. 2004 Jul;86(4):172-8.

5 Crowley NJ, Seigler HF. Late recurrence of malignant melanoma. Analysis of $168 \mathrm{pa}-$ tients. Ann Surg. 1990 Aug;212(2):173-7.

6 Cruz J, Reis-Filho JS, Lopes JM. Primary cutaneous malignant melanoma with lipoblastlike cells. Arch Pathol Lab Med. 2003 Mar; 127(3):370-1.

7 Bhaijee F, Jackson MS, Lewin JR, Rezeanu L. Metastatic melanoma with lipoblast-like and signet ring cell morphology: an unusual morphologic variant of a protean malignancy. Am J Clin Pathol. 2013;140 suppl 1:A30.
8 Iwamoto S, Burrows RC, Kalina RE, George $\mathrm{D}$, Boehm M, Bothwell MA, et al. Immunophenotypic differences between uveal and cutaneous melanomas. Arch Ophthalmol. 2002 Apr;120(4):466-70.

9 McLean IW, Foster WD, Zimmerman LE, Gamel JW. Modifications of Callender's classification of uveal melanoma at the Armed Forces Institute of Pathology. Am J Ophthalmol. 1983 Oct;96(4):502-9.

10 Messmer E, Hoops JP, Stefani FH. Signet-ring cell malignant melanoma of the choroid. Invest Ophthalmol Vis Sci. 1996;37:S244.

11 Kocovski L, Alowami S. Signet-ring cell melanoma: a potential diagnostic pitfall. Am J Dermatopathol. 2014 Dec;36(12):985-8.

12 Gaynor R, Irie R, Morton D, Herschman HR. S100 protein is present in cultured human malignant melanomas. Nature. $1980 \mathrm{Jul}$; 286(5771):400-1.
13 Ordóñez NG. Value of melanocytic-associated immunohistochemical markers in the diagnosis of malignant melanoma: a review and update. Hum Pathol. 2014 Feb;45(2):191205

14 Yavuzyigitoglu S, Kilic E, Vaarwater J, de Klein A, Paridaens D, Verdijk RM; Rotterdam Ocular Melanoma Study Group (ROMS). Lipomatous Change in Uveal Melanoma: Histopathological, Immunohistochemical and Cytogenetic Analysis. Ocul Oncol Pathol. 2016 Apr;2(3):133-5.

15 Nonaka D, Chiriboga L, Rubin BP. Sox10: a pan-schwannian and melanocytic marker Am J Surg Pathol. 2008 Sep;32(9):1291-8. 